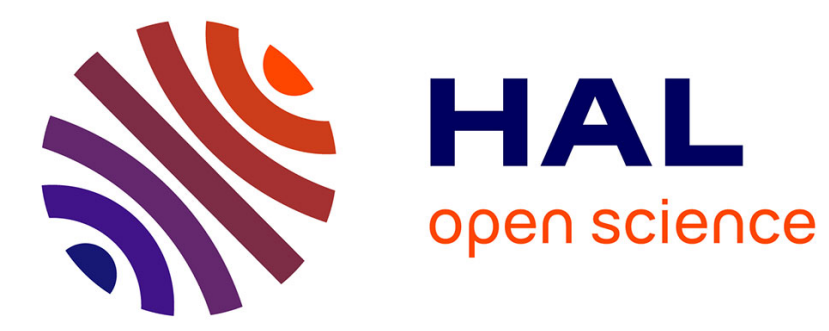

\title{
Achievement of avian influenza virus-like particles that could be used as a subunit vaccine against low pathogenic avian influenza strains in ducks
}

\author{
Anne Prel, Ghislaine Le Gall-Recule, Véronique Jestin
}

\section{- To cite this version:}

Anne Prel, Ghislaine Le Gall-Recule, Véronique Jestin. Achievement of avian influenza virus-like particles that could be used as a subunit vaccine against low pathogenic avian influenza strains in ducks. Avian Pathology, 2008, 37 (05), pp.513-520. 10.1080/03079450802357001 . hal-00540132

\section{HAL Id: hal-00540132 \\ https://hal.science/hal-00540132}

Submitted on 26 Nov 2010

HAL is a multi-disciplinary open access archive for the deposit and dissemination of scientific research documents, whether they are published or not. The documents may come from teaching and research institutions in France or abroad, or from public or private research centers.
L'archive ouverte pluridisciplinaire HAL, est destinée au dépôt et à la diffusion de documents scientifiques de niveau recherche, publiés ou non, émanant des établissements d'enseignement et de recherche français ou étrangers, des laboratoires publics ou privés. 


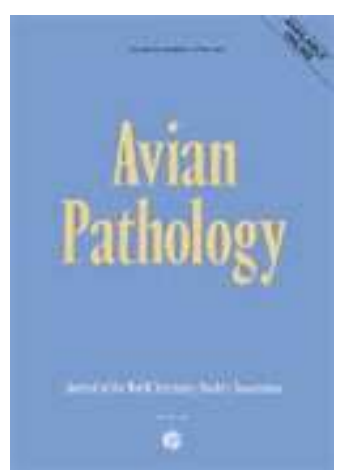

Achievement of avian influenza virus-like particles that could be used as a subunit vaccine against low pathogenic avian influenza strains in ducks

\begin{tabular}{|c|c|}
\hline Journal: & Avian Pathology \\
\hline Manuscript ID: & CAVP-2008-0085 \\
\hline Manuscript Type: & Original Research Paper \\
\hline $\begin{array}{r}\text { Date Submitted by the } \\
\text { Author: }\end{array}$ & 03-Jun-2008 \\
\hline Complete List of Authors: & $\begin{array}{l}\text { Prel, Anne; AFSSA, UVIPAC } \\
\text { Le Gall-Recule, Ghislaine; AFSSA, UVIPAC } \\
\text { Jestin, Véronique; AFSSA }\end{array}$ \\
\hline Keywords: & $\begin{array}{l}\text { Avian influenza, virus-like particles, duck, baculovirus expressed } \\
\text { proteins }\end{array}$ \\
\hline
\end{tabular}

\section{SCHOLARONE ${ }^{\text {TH }}$ Manuscripts}


Achievement of avian influenza virus-like particles that could be used as a subunit vaccine against low pathogenic avian influenza strains in ducks

\section{Anne Prel, Ghislaine Le Gall-Reculé* and Véronique Jestin}

French Agency for Food Safety (AFSSA), Avian and Rabbit Virology, Immunology and Parasitology Unit, French Reference Laboratory for Avian Influenza and Newcastle Disease, B.P. 53, 22440 Ploufragan, France.

Running title: Avian influenza virus-like particles

*To whom correspondence should be addressed.

Tel. +332960162 98. Fax: +332960162 63. E-mail g.legall@afssa.fr

Received 3 June 2008: 
1 Abstract

3 Infections with H5/H7 low pathogenic avian influenza (LPAI) viruses are now notifiable

4 because such viruses can mutate into highly pathogenic avian influenza (HPAI) viruses,

5 leading to serious problems for both animal and public health. Domestic ducks can play a

6 crucial role in the transmission of H5 LPAI viruses to other poultry. Although prime boost

7 vaccination using, respectively, a recombinant and an inactivated vaccine was shown to be

8 protective in ducks against H5N1 HPAI, vaccination of domestic ducks against H5 LPAIV

9 is poorly documented. However, substituting inactivated vaccines with subunit vaccines

10 might be more advantageous. In this context, we generated a triple recombinant

11 baculovirus composed of HA and NA proteins derived from a French H5N3 LPAI virus

12 strain and the M protein derived from an Italian H7N1 LPAI virus strain. We describe a

13 molecular construction strategy that enabled the development of virus-like particles

14 (VLPs). Western blot analyses and neuraminidase inhibition assay of cell supernatants

15 purified by sucrose density gradient ultracentrifugation showed that HA, NA and M1

16 proteins were expressed and co-released. Electron microscopy examination revealed VLPs

17 that were morphologically identical to wild-type virus. Immunogold electron microscopy

18 demonstrated that $\mathrm{H} 5$ and $\mathrm{N} 3$ proteins were present on the VLP surface, and

19 haemagglutination and neuraminidase assays showed that the $\mathrm{H}$ and $\mathrm{N}$ proteins,

20 respectively, were biologically active. In addition, VLP immunogenicity (induction of

21 haemagglutination-inhibiting antibodies) was demonstrated in SPF Muscovy ducks.

22 According to our successful previous experimental results of protection in ducks following

23 vaccination with the three baculovirus-expressed proteins, the present results make feasible

24 the reliable use of H5N3 VLPs as a subunit vaccine in this species. 


\section{Introduction}

27 Avian influenza (AI) remains an economic threat to commercial poultry worldwide. Since 28 1997, direct avian-to-human transmission of lethal AI viruses of the H5 subtype has 29 elevated the need to control AI beyond economic considerations. Domestic ducks can play 30 crucial roles in the transmission of H5 low pathogenic (LP) (Cherbonnel et al., 2007) and 31 highly pathogenic (HP) avian influenza viruses (Sturm-Ramirez et al., 2005), and a few inactivated and recombinant commercial or experimental vaccines have been assessed experimentally in these species essentially for the prevention of H5N1 HP (Middleton et al., 2007; Steensels et al., 2007). However, in the field the protection of vaccinated ducks remains questionable as suggested by the reoccurrence of H5N1 HP in Vietnam despite massive vaccination (OIE). Few data have been reported concerning the protection

37 afforded by inactivated vaccines against H5 LPAI infection in ducks (Prel et al., 2007).

38 However, alternative vaccines may increase this protection. Recombinant avian influenza

39 vaccines such as fowlpox virus-based AI vaccines cannot be used in prime-boost because a 40 strong immunity may be directed against the vector (Swayne et al., 2000). This is the 41 reason why, in a recent study, a boost with an inactivated AI vaccine was performed after a 42 prime with a fowlpox virus-based AI vaccine (van den Berg et al., 2008). Although the 43 latter schedule was shown to be efficient in a HPAI challenge model, it might be more 44 advantageous to substitute inactivated vaccines with subunit vaccines in order to better differentiate vaccinated birds from infected ones by testing antibodies against other influenza proteins that would not be included in the subunit vaccine (Differentiating

47 Infected from Vaccinated Animals, DIVA, strategy). Vaccines composed of virus-like 48 particles (VLPs) have been generated for a variety of viruses (Noad \& Roy, 2003) and 49 have demonstrated a great potential for the prevention of infectious diseases. Indeed VLPs 
were shown to be very immunogenic. In addition, humoral, cell-mediated, and mucosal immune responses have been reported in sheep following immunization with bluetongue virus VLPs (Roy, 1996). The strategy using the immunogenic properties of avian influenza virus surface glycoproteins seems accessible since Latham \& Galarza (2001) showed the possibility of producing VLPs using a quadruple recombinant baculovirus encoding hemagglutinin and neuraminidase surface glycoproteins and M1 and M2 matrix proteins of H3N2 human influenza virus. Pushko et al. (2005) confirmed this approach by producing VLPs using a triple recombinant baculovirus encoding HA, NA, and M1 matrix of an avian influenza virus H9N2 isolated from children. They also showed that immunization of mice with these VLPs reduced challenge virus replication and conferred a protection against a homologous influenza virus challenge. Although recombinant HA protein has been shown to be immunogenic in poultry (Crawford et al., 1999), no data are available regarding VLPs in this species. If avian influenza virus VLP immunogenicity were to be shown in ducks, VLPs could provide a solution to the drawbacks of inactivated vaccines.

In this context, we previously attempted to develop a H5N3 avian influenza VLP vaccine expressed from insect cells, comprising the two structural proteins HA and NA derived from a recent French LPAI virus strain A/Duck/France/02166/2002 (H5N3) and the M matrix protein derived from an Italian LPAI virus strain A/Chicken/Italy/1067/99 (H7N1) (Prel et al., 2007). We generated a triple recombinant baculovirus and we demonstrated that the three proteins were successfully expressed in infected Sf9 insect cells and displayed the expected biological activity. Although we showed that the three proteins were co-released in cell media, ours results in electron microscopy did not display the formation of VLPs. One of the proposed explanations was that one protein might have been expressed in a too limiting quantity that could decrease the yield of VLP released from Sf9 cells. Indeed, the achievement of appropriate expression levels of each protein 
75 represents one of the difficulties in making complex VLPs (van Oers, 2006). In this 76 previous work, the HA and NA proteins were both expressed under the control of the 77 polyhedrin promoter. After several amplifications, the HA gene could be excluded by recombination between the two promoters.

79 In this study, we have changed the molecular construction strategy and have achieved 80 a novel triple recombinant plasmid that allowed us to optimise and finalize the 81 development of an H5N3 avian influenza VLP vaccine for ducks.

\section{Materials and Methods}

86 Generation of a triple recombinant baculovirus. We used the double recombinant

87 baculovirus plasmid pMH5 previously generated by our team (Prel et al., 2007). In this construction, each gene was located within its own expression cassette that included either the polyhedrin or the p10 promoter and transcription termination sequences. The HA

90 protein (GenBank accession number: AJ632268) was produced under the control of the

91 polyhedrin promoter whereas the M protein (GenBank accession number: AJ416630) was 92 produced under the control of the promoter $\mathrm{p} 10$. A full-length cDNA copy of the NA gene 93 of A/Duck/France/02166/2002 (H5N3) strain (GenBank accession number: AJ849934) 94 was cloned downstream of the p10 promoter into pFastBac Dual vector (Invitrogen) at the 95 XhoI and NheI sites to generate a pN3-2 recombinant plasmid. Then, using a subcloning strategy, the "p10 promoter, N3 gene and HSV TK termination sequence" was inserted into

97 a pMH5 recombinant plasmid at the BstZ17I and AvrII sites to generate the pMH5N3-2 recombinant plasmid (Figure 1). The NA gene and the junctions were sequenced to check

99 that no mutation had been introduced during cloning. 

vector) and helper plasmid were used to generate recombinant bacmid according to the

102 manufacturer's instructions. Confluent Spodoptera frugiperda (Sf9) insect cells seeded in a

$10325 \mathrm{~cm}^{2}$ flask at $2 \times 10^{6}$ cells were transfected with a mixture of $1 \mu \mathrm{g}$ of bMH5N3-2 bacmid

$104(15 \mu \mathrm{l})$ and $15 \mu \mathrm{l}$ of Cellfectin reagent (Invitrogen). The supernatant was harvested 5 days

105 after transfection. An amplification of virus stock was performed to obtain a higher titre.

106 The infectious titre of the recombinant baculovirus stock was determined by a plaque assay

107 using Sf9 cells.

108

109 Indirect immunofluorescence examination of infected Sf9 cells. Sf9 cells were seeded

110 in 96 microwell plates at a cell density of $1 \times 10^{5}$ cells/well, and were infected with the

111 triple recombinant baculovirus at an MOI of 5. At $72 \mathrm{~h}$ post infection, Sf9 cells were fixed

112 in methanol-acetone. Fifty $\mu \mathrm{l}$ of SPF chicken serum (1:10 dilution) were dispensed in two

113 wells as negative controls. To examine the expression of H5, N3, and M1 proteins

114 respectively, $50 \mu 1$ of either chicken-specific $\mathrm{H} 5$ or N3 serum (1:10 dilution) or $50 \mu \mathrm{l}$ of

115 M1 murine monoclonal antibody (ATCC HB 64) (1:100 dilution) were dispensed in 116 duplicate in the other wells. Chicken-specific anti-H5 serum and anti-N3 serum were 117 prepared from SPF chickens that had received three inoculations of either $100 \mu \mathrm{g}$ pcDNA118 H5 plasmid (Prel et al., 2007) or $100 \mu \mathrm{g}$ pcDNA-N3 plasmid (Prel et al., 2007) in $100 \mu \mathrm{l}$ 119 with the Medijector device (Antares pharma, Inc, Minneapolis, MN), respectively. The 120 plate was incubated at $37^{\circ} \mathrm{C}$ for $45 \mathrm{~min}$. At the end of incubation, the wells were washed 121 three times for $5 \mathrm{~min}$ in $200 \mu \mathrm{l}$ PBS. Then, $50 \mu \mathrm{l}$ of either rabbit anti-chicken FITC122 conjugated antibody (Nordic, 1:36 dilution) or goat anti-mouse FITC-conjugated antibody 123 (Rockland, 1:500 dilution) and Evans Blue (1:75 working dilution) were added in each 124 well and incubated at $37^{\circ} \mathrm{C}$ for $45 \mathrm{~min}$. After incubation, wells were washed three times 
125 for $5 \mathrm{~min}$ in PBS. Wells were overlaid with $200 \mu \mathrm{l}$ of PBS/glycerol (v/v) and read under an

126 UV microscope (Olympus, IMT 2). Uninfected cells were used as negative controls and

127 were treated with the different sera as infected cells.

129 Purification of influenza VLPs. To examine whether the recombinant proteins could self-

130 assemble into VLPs, Sf9 insect cells $\left(35 \times 10^{6}\right.$ cells/flask of $\left.175 \mathrm{~cm}^{2}\right)$ were infected with

131 triple recombinant baculoviruses at an MOI of 5. Three days post infection, infected cells

132 and culture supernatants were harvested and clarified by centrifugation for 30 min at 2,000

$133 g$ at $4{ }^{\circ} \mathrm{C}$ to remove cell debris. VLPs in supernatants were pelleted by ultracentrifugation 134 at $130,000 \mathrm{~g}$ at $4{ }^{\circ} \mathrm{C}$ for $3 \mathrm{~h}$.

135 To assess protein expression, the sedimented particles were resuspended in $2 \mathrm{ml}$ of 136 phosphate-buffered saline (PBS) at $4{ }^{\circ} \mathrm{C}$ overnight, then loaded onto a $15-55 \%$ (w/w) 137 continuous sucrose density gradient and sedimented by ultracentifugation at 130,000 $\mathrm{g}$ at $1384^{\circ} \mathrm{C}$ for $16 \mathrm{~h}$. Fractions of $1 \mathrm{ml}$ were collected from the top of the sucrose gradient, washed 139 by dilution in PBS and sedimented by ultracentrifugation at $195,000 \mathrm{~g}$ at $4{ }^{\circ} \mathrm{C}$ for $1 \mathrm{~h}$. The 140 pellets were resuspended in $200 \mu \mathrm{l}$ of PBS and stored at $-70{ }^{\circ} \mathrm{C}$.

141 For electron microscopy and haemagglutination activity, the sedimented particles were 142 purified through a $20-30-50 \%$ sucrose density cushion at $130,000 \mathrm{~g}$ at $4{ }^{\circ} \mathrm{C}$ for $1.75 \mathrm{~h}$. The 143 VLP band was collected at the $30-50 \%$ interface and washed with PBS. VLPs were 144 pelleted by ultracentrifugation and resuspended in $60 \mu 1$ of PBS.

145

146 Protein expression. HA and M1 protein expression in each sucrose fraction was analysed 147 by western blotting. The proteins $(20 \mu \mathrm{l})$ from sucrose-gradient purified VLPs were 148 separated by sodium dodecylsuphate polyacrylamide gel electrophoresis (SDS-PAGE) 149 using 10-20\% gradient polyacrylamide gels (Biorad), and transferred onto PVDF 
membranes (Polyvinylidene fluoride, Amersham). HA protein was identified using specific-pathogen free (SPF) chicken specific serum H5N3/02166 and a peroxidaselabelled rabbit anti-chicken IgG conjugate (Sigma). M1 protein was identified using M1murine monoclonal antibody (ATCC HB 64) and a peroxidase-labeled goat anti-mouse IgG conjugate (Sigma). NA protein expression and its activity were analysed by neuraminidase inhibition assay using fetuin as substrate for the cleavage of sialic acid. In this test, the NA antibodies present in the serum inhibited the action of the viral enzyme on the fetuin substrate. An aliquot of each sucrose fraction was incubated with chicken $\mathrm{H} 2 \mathrm{~N} 3$ antiserum for $1 \mathrm{~h}$ at room temperature. Then, the neuraminidase inhibition assay was performed as described by van Deusen et al. (1983).

To estimate the efficacy of the purification method and the ratio of the three proteins in the VLPs, $10 \mu \mathrm{l}$ of the $30-50 \%$ sucrose interface purified VLPs was analysed by Coomassie blue-stained 10\% SDS-PAGE. Otherwise, haemagglutination activity of VLPs was tested using a haemagglutination assay according to international standards (O.I.E., 2004). Two-fold serial dilutions of $25 \mu \mathrm{l}$ of $30-50 \%$ sucrose interface purified VLPs in phosphate-buffered saline were prepared and incubated at room temperature for $30 \mathrm{~min}$ with $50 \mu 1$ of chicken blood cells. The haemagglutination was inspected visually.

Electron microscopy. For negative staining of VLPs, $20 \mu \mathrm{l}$ of 30-50\% sucrose interface purified VLPs were incubated with chicken-specific serum H5N3/02166 (1:10 dilution) at room temperature for $30 \mathrm{~min}$. Then, the sample was adsorbed onto a carbon- and formvarcoated copper grid for $30 \mathrm{~min}$. The excess VLP suspension was removed by blotting with filter paper. The grid was negatively stained with $2 \%$ phosphotungstic acid (pH 6.45) for 2 min, air-dried, and examined with a transmission electron microscope (JEOL). 
175 Immunogold electron microscopy. Twenty $\mu 1$ of $30-50 \%$ sucrose interface purified VLPs were incubated with chicken-specific anti-H5 or anti-N3 serum (1:10 dilution) for 30 min at room temperature. The sample was adsorbed onto a carbon- and formvar-coated grid for $30 \mathrm{~min}$ and washed three times in PBS. The grids were exposed to $5 \mathrm{~nm}$ gold particlelabelled rabbit anti-chicken IgG (Sigma) diluted in PBS (1:30) for $30 \mathrm{~min}$ at room temperature, and washed three times in PBS, 5 min each. Finally, the grids were negatively stained with $2 \%$ phosphotungstic acid ( $\mathrm{pH}$ 6.45) for $2 \mathrm{~min}$, air dried, and examined with a transmission electron microscope (JEOL).

Immunisation of ducks and haemagglutinin inhibition (HI) test. For large scale production of VLPs for inoculation of ducks, Sf9 cells were grown in suspension in two $250 \mathrm{ml}$ spinner flasks at a density of about $1.0 \times 10^{6}$ cells $/ \mathrm{ml}$. They were infected with triple recombinant baculovirus at an MOI of 4 and harvested $90 \mathrm{~h}$ post infection. Cell supernatants were purified through a $20-30-50 \%$ sucrose density as previously described. VLPs were resuspended in $180 \mu \mathrm{l}$ of PBS. Protein concentration of the purified fraction was determined by using a colorimetric assay (MicroBC Assay Protein Quantification Kit, Uptima) with bovine serum albumin as the standard. Fifteen 3.5-week-old specificpathogen-free (SPF) Muscovy ducks (Cairina moschata domesticus) from our institute, split into 3 groups of 5 ducks each, were housed in a room in our BSL2+ experimental facilities with filtered air. The first group was used as an SPF control and ducks remained unvaccinated. Ducks of the second and the third groups were inoculated subcutaneously three weeks apart with $20 \mu \mathrm{g}$ and $2 \mu \mathrm{g}$ (total protein per dose), respectively, of purified VLPs formulated with an oily adjuvant (kindly provided by Merial), in a final volume of 1 $\mathrm{ml} /$ duck. Blood samples were collected from 5 ducks at random before immunisation, then 
199 in all ducks just before boost, and when the ducks were killed three 3 weeks after the

200 boost.

201 To assess HA responses, every serum sample was analysed by HI test. HI tests were 202 performed according to international standards (OIE, 2004) using 4 haemagglutinin units 203 of inactivated A/duck/France/02166/2002(H5N3) AI as antigen. Antibody titres were 204 expressed as $\log _{2}$ of the highest dilution giving $100 \%$ inhibition of the haemagglutination. 205 The threshold was defined as $\geq 4 \log _{2}$.

206

208 Results

210 Expression of recombinant proteins in insect cells. In order to generate H5N3 VLPs in

211 Sf9 insect cells, HA and NA genes derived from a recent French low-pathogenic avian 212 influenza A/Duck/France/02166/2002 (H5N3) virus strain and the M gene derived from an 213 Italian A/Chicken/Italy/1067/1999 (H7N1) virus strain were introduced into a baculovirus 214 shuttle vector to obtain a triple recombinant plasmid pMH5N3-2. In our previous study 215 (Prel et al., 2007), HA and NA proteins were both expressed under the transcriptional 216 control of the polyhedrin promoter. In the present study, the NA gene was inserted in the 217 same orientation under the control of the p10 promoter in order to avoid eventual 218 competition between HA and NA genes. The $\mathrm{M}$ gene was inserted in the opposite 219 orientation under the control of the p10 promoter (Figure 1).

220 To evaluate the expression of HA, NA, and M1 proteins in Sf9 insect cells, the pellets 221 of insect cells infected with this triple recombinant baculovirus were analysed by western 222 blot and neuraminidase inhibition assay. As previously reported using the first baculovirus construct (Prel et al., 2007), we could check that the three proteins were efficiently 
224 expressed in Sf9 cells following infection with the new triple recombinant baculovirus construct (data not shown). To localize the subcellular distribution of the expressed

226 proteins, we performed indirect immunofluorescence analyses of Sf9 cells infected with bMH5N3-2. Observations confirmed the expression of all three proteins. Staining

228 experiments with anti-H5 and anti-N3 antibodies showed that HA and NA expressed 229 proteins were localized at the level of the cell membrane (Figure 2), as previously shown 230 with recombinant baculoviruses expressing human influenza HA and NA proteins (Latham \& Galarza, 2001). Staining experiments with anti-M1 antibodies showed that the M1

232 expressed protein was localized at the level of the cell membrane, although the 233 fluorescence intensity was poor (Figure 2).

Influenza VLPs were purified from culture media $72 \mathrm{~h}$ post-infection by $15-55 \%$ continuous sucrose gradient centrifugation. Seven fractions (sucrose densities: $26.9 \%$ to $52.7 \%$ ) were analysed by western blotting for HA and M1 expressions. The same fractions were assessed by a neuraminidase inhibition assay for NA expression and activity.

As shown in Figure 3A, the majority of HA protein (66 to $68 \mathrm{kD}$, approximately) was detected in fractions 7 to 11 corresponding to sucrose densities of $33.1 \%$ to $49.2 \%$. The

240 HA electrophoretic migration pattern differed from native HA (75 kD) synthesized during virus multiplication on embryonated-eggs, presumably reflecting glycosylation differences

242 between avian and insect cells. We observed the same HA electrophoretic migration pattern for the HA protein expressed in Sf9 mimic insect cells (Invitrogen) which have

244 been modified to greatly enhance the production of complex sialylated N-linked glycans to express recombinant proteins like those found in mammalian systems (data not shown).

246 Regarding the M1 protein $(29 \mathrm{kD})$, the majority was detected in fractions 8 to 11 corresponding to sucrose densities of 36.5 to $49.2 \%$ (Figure 3B). Western blot analyses revealed that HA and M1 protein expressions were lower in fraction 9 (Figure 3, A and B) 
249 but this observation was correlated with a lower protein concentration in this fraction. The

250 NA protein and NA activity were detected in the 7 to 11 fractions (Figure 3C). The yield of

251 VLPs production in the whole of the 7 to 11 sucrose fractions was estimated to be about

$2521.4 \mu \mathrm{g}$ per $\mathrm{ml}$ of Sf9 cell culture by using a colorimetric assay.

253 All these results demonstrated that insect cells infected with triple recombinant 254 baculoviruses co-expressed the three proteins. These were co-released in the culture 255 medium and were all present at the same sucrose densities of $36.5 \%$ to $49.2 \%$, indicating that HA, NA, and M1 proteins could self-assemble into VLPs.

Evaluation of influenza VLPs by electron microscopy and by Coomassie blue-stained

259 SDS-PAGE. In order to check whether these proteins were released from cells into culture medium as VLPs that budded from the cell surface, concentrated supernatant was purified on $20-30-50 \%$ sucrose density cushions. Influenza VLPs were stopped by $50 \%$ sucrose cushion while baculovirus passed through (Pushko et al., 2005). The 30-50\% interface band was analysed by electronic microscopy and Coomassie blue-stained SDS-PAGE. presence of a great number of VLPs. These particles were morphologically identical to the wild-type virus. Indeed, there were spherical-shaped particles with a diameter of approximately 80 to $120 \mathrm{~nm}$, with surface spikes (Figure 4). In the 20-30\% sucrose interface, we observed membrane parts that confirmed western blotting results for which, only HA and NA proteins gave a positive signal at sucrose density fractions of $33.1 \%$.

Coomassie blue-stained SDS-PAGE of $30-50 \%$ sucrose interface purified VLPs 271 showed the good purity of the fraction containing VLPs (Figure 5). The 3 proteins were visualized and we estimated the HA and NA protein ratio in particles to be one NA for at 
273 least four HA, which is very close to the theoretical ratio of 5 to 1 , whereas the relative

274 amount of the M1 protein was low.

276 Immunogold electron microscopy and haemagglutination activity of VLPs. We

277 investigated the protein composition of the spikes that protruded from the surface of the

278 VLPs using immunogold electron microscopy. VLPs incubated first with an anti-HA

279 serum, then with a gold-labelled anti-chicken conjugate, were displayed by transmission

280 electron microscopy. The examination revealed gold particles on the surface of VLPs and

281 demonstrated the presence of HA proteins (Figure 6A). Similarly, immunogold labelling

282 with the anti-NA antibody and electron microscopy revealed the presence of NA

283 glycoproteins on the surface of VLPs (Figure 6B).

284 Regarding haemagglutination activity of VLPs, the assay revealed a high activity 285 (1:256 dilution) and demonstrated that the HA proteins present on the surface of VLPs 286 were biologically active.

288 Immunogenicity in ducks. The immunity induced by the VLPs was checked in SPF 289 Muscovy ducks. As expected, no H5-specific serum antibody was detected in ducks before 290 vaccination or in unvaccinated animals during the assay. After the prime inoculation all 291 ducks developed HI titres that increased after the boost. After the boost and regardless of 292 the VLP quantity inoculated, all the ducks exhibited positive HI titres $\left(\geq 4 \log _{2}\right)$ (Table 1$)$

293 Geometric means of HI titres of both inoculated groups were almost similar, indicating that

294 a 10-fold quantity of VLP did not enhance the serum antibody response. In addition, the 295 standard deviations were very low whatever the tested dose, underlying no significant 296 individual variation in the antibody response. 
299 Discussion

300

301 Since H5 LP avian influenza viruses can mutate into HPAI viruses, it is necessary to

302 prevent their introduction and spread in poultry because such infections can be the source

303 of serious epizootics that could have public health consequences. Recombinant vaccines

304 have been generated and evaluated for ducks. They were used in combination with

305 inactivated vaccines (van den Berg et al., 2008). Since influenza VLPs produced by

306 recombinant baculoviruses emerged as promising candidates for influenza vaccines

307 (Latham \& Galarza, 2001), we thought that substituting inactivated vaccines by subunit

308 vaccines as VLPs might be more advantageous, notably regarding the ability to easily

309 differentiate vaccinated birds from infected ones. In addition, Bright et al. (2007)

310 confirmed that human influenza VLPs elicited broader reactive immune responses than

311 baculovirus-produced recombinant HA. Regarding the strategy used to produce influenza

312 VLPs, when we initiated this work, several models were described that differed on the type

313 of viral proteins required. Latham \& Galarza (2001) demonstrated that the expression of

314 four structural proteins (HA, NA, M1, and M2) was sufficient for the formation and the

315 release of VLPs, whereas one other team (Pushko et al., 2005; Pushko et al., 2007; Bright

316 et al., 2007) described the development of H9N2 or H3N2 influenza VLPs comprised of

317 only three proteins (HA, NA, and M1). Another study reported the assembly and the

318 release into cell culture supernatant of VLPs containing only HA and M1 proteins

319 following co-infection with two individual recombinant baculoviruses (Galarza et al.,

320 2005). This approach was recently used by Quan et al. (2007) but not by Galarza's team

321 that used again quadruple recombinant baculovirus proteins to prepare a VLP vaccine

322 aimed to protect against infection with the H1N1 1918 influenza virus (Matassov et al., 
323 2007). The various models of avian influenza VLPs that have been developed to date have

324 always included the matrix protein M1. This protein, which is the most abundant in the 325 virus particle, was shown to be critically important for viral morphogenesis and budding (Lohmeyer et al., 1979; Roberts et al., 1998). Studies of VLP formation have demonstrated that the assembly, budding, and release process was driven solely by the expression of the M1 protein (Gomez-Puertas et al., 2000). However, a recent study seems to refute this. Chen et al. (2007) developed a noncytotoxic plasmid-based VLP system and showed that M1 was not required to produce VLPs morphologically similar to authentic virions. They revealed that influenza particle budding is not driven by M1 but is rather dependent on the expression of cytoplasmic tails of the two proteins HA and NA. However, they confirmed that M1 is required to produce an infectious VLP. Currently, no data are available to confirm that VLPs comprised of only HA and NA proteins could be developed. not experimentally demonstrate the role of the M2 protein in VLPs (Latham \& Galarza, 2001), we chose to construct a triple recombinant baculovirus plasmid encoding HA, NA, and M1 proteins. In the context of production of an avian subunit vaccine, it was interesting to exploit the immunogenicity of the NA protein besides the HA one. Indeed, Brett \& Johansson (2005) showed that immunization of mice with both baculovirusexpressed HA and NA proteins resulted in a broader immune response and induced a greater reduction in viral replication following a viral challenge by a distantly related heterologous influenza strain, in contrast to the protection afforded by immunization based on the HA protein alone. These results suggest that a vaccine containing the NA protein tolerates more antigenic drift (van Oers, 2006).

We have followed the strategy used by Latham \& Galarza (2001) and Pushko et al.

347 (2005) that consists of expressing several proteins simultaneously using one recombinant 
baculovirus instead of several individual ones. Indeed, the achievement of VLPs following either co-infection of cells with three single recombinant baculoviruses HA, NA and M1, or co-infection of cells with a double recombinant baculovirus HA-M1 and a single recombinant baculovirus NA, was shown to be inconclusive (Pushko et al., 2005). In a first approach, we constructed a triple recombinant plasmid by subcloning the expression cassette "polyhedrin promoter, N3 gene and HSV tk termination sequence" of the recombinant baculovirus plasmid pNA downstream the HA gene into the double recombinant plasmid pMH5 previously generated, resulting in a pMH5N3 plasmid (Prel et al., 2007). Thus in this approach, HA and NA proteins were both expressed in the same direction under the control of the polyhedrin promoter, according to the strategy followed by Pushko et al. (2005). Although Prel et al. (2007) showed that the three proteins were co-released into the cell supernatant, electron microscopy did not reveal the presence of VLPs, suggesting that either the number of VLPs generated might have been too low to be detected by electron microscopy or no VLPs had produced.

Compared to the strategy followed by Pushko et al. (2005), we have kept HA and NA genes in the same orientation but have changed the molecular construction by expressing each protein under the transcriptional control of two different promoters to avoid eventual competition between the two genes. Western blots and neuraminidase inhibition assay indicated that all three proteins were expressed in infected Sf9 insect cells and were localized at the level of the cell membrane, as expected (Latham \& Galarza, 2001). Electron microscopy results showed that these proteins were assembled and budded from insect cells to be released as virus-like particles in the culture medium and resembled influenza virus particles in morphology and size. VLPs bore surface spikes that were anchored in the membrane of the particles and were projected outward as HA and NA 
372 proteins, as shown by immunogold labeling and electron microscopy. In addition, VLPs

373 exhibited haemagglutination and neuraminidase activities.

374 The experimental assay performed in SPF Muscovy ducks showed that inoculation of

375 VLPs induced a positive HA antibody response for all the VLP-inoculated animals that 376 confirm the biological activity of the VLPs and their immunogenicity in ducks. The levels of H5 antibodies elicited by the 2 tested VLP doses were similar suggesting that 378 vaccination with as little as $2 \mu \mathrm{g}$ of total protein is sufficient to induce a significant immune response. In our previous study, we immunized specific pathogen free Muscovy

380 ducks with lysates of Sf9 cells infected with a triple recombinant baculovirus (bMH5N3) to assess protein immunogenicity and protection afforded following a homologous A/Duck/France/02166/2002 (H5N3) virus challenge (Prel et al., 2007). We showed that the three baculovirus-expressed proteins were immunogenic. The ducks of the vaccinated group exhibited a geometric mean of $5.9 \log _{2}$ titres using $\mathrm{HI}$ test and after challenge, the vaccination afforded a significant decrease of cloacal shedding and a delayed peak of tracheal shedding. In the present study, we demonstrate that VLPs induce HI titres similar to those shown as being protective in ducks. Although further experiments in ducks will be necessary to definitely demonstrate the protective immune response induced by the VLPs, given our previous results of protection, the functional properties of the HA and NA proteins presently expressed as VLPs, and the immunogenicity of VLPs in ducks, we are optimistic. A trial is scheduled as soon as is available the recombinant vaccine intended for ducks and currently in preparation through the European project NOVADUCK. Indeed, we target the use of our VLPs for boost after priming with the recombinant vaccine. VLPs should allow the development of safe and effective subunit vaccines to control the spread of LPAI in ducks. VLPs are more easily purified than recombinant proteins and 
often give strong immune reaction (van Oers, 2006). Moreover, according to the

398 epidemiological context, it would be easier to replace H5 or/and N3 gene from the triple-

400

401

402

403

404

405

406

407

\section{Acknowledgement}

409

410 The authors are grateful to Anne-Cécile Nignol, from the Virus Genetics and Biosecurity

411 unit at AFSSA, for insect cell cultures. They thank Eric Niqueux, Carole Guillemoto, and

412 Marie-Odile Le Bras from the unit for their assistance in purification of VLPs and

413 serological analyses, as well as the personal of the Service of Breeding and

414 Experimentation in Avian Pathology of AFSSA. They wish to acknowledge Elisabeth

415 Reperant for the review of English expression. This work was partially funded by the

416 European REX Epizone.

417

418

419 References

420
Deleted: Brett, I.C. \& Johansson, B.E. (2005). Immunization against influenza A virus: comparison of conventional inactivated, live-attenuated and recombinant baculovirus produced purified hemagglutinin and

neuraminidase vaccines in a murine

model system. Virology 339, 273-280.II

Bright, R.A., Carter, D.M., Crevar, C.J.,

Toapanta, F.R., Steckbeck, J.D., Cole,

K.S., Kumar, N.M., Pushko, P., Smith,

G., Tumpey, T.M. \& Ross, T.M. (2008)

Cross-Clade Protective Immune

Responses to Influenza Viruses with

H5N1 HA and NA Elicited by an

Influenza Virus-Like Particle. PLOS ONE 3, e1501.प[

Bright, R.A., Carter, D.M., Daniluk, S.,

Toapanta, F.R., Ahmad, A., Gavrilov, V.,

Massare, M., Pushko, P., Mytle, N.,

Rowe, T., Smith, G. \& Ross, T.M.

(2007). Influenza virus-like particles

elicit broader immune responses than

whole virion inactivated influenza virus

or recombinant hemagglutinin. Vaccine

25, 3871-3878.II

Chen, B.J., Leser, G.P., Morita, E. \&

Lamb R.A. (2007). Influenza virus

hemagglutinin and neuraminidase, but not

the matrix protein, are required for

assembly and budding of plasmid-derived

virus-like particles. Journal of Virology

81,7111-7123. II

Cherbonnel, M., Lamande, J., Allee, C.,

Schmitz, A., Ogor, K., Le Gall-Reculé,

G., Le Bras, M.O., Guillemoto, C., Pierre,

I., Picault, J.P. \& Jestin, V. (2007).

Virologic findings in selected free-range

mule duck farms at high risk for avian

influenza infection. Avian Diseases 51,

408-413.II

Crawford, J., Wilkinson, B.

Vosnesensky, A., Smith, G., Garcia, M., Stone, H. \& Perdue, M.L. (1999).

Baculovirus-derived hemagglutinin

vaccines protect against lethal influenza

infections by avian $\mathrm{H} 5$ and $\mathrm{H} 7$ subtypes.

Vaccine 17, 2265-2274.

Galarza, J.M., Latham, T. \& Cupo, A

(2005). Virus-like particle (VLP) vaccine

conferred complete protection against a

lethal influenza virus challenge. Viral

Immunology 18, 244-251.II

Gomez-Puertas, P., Albo, C., Perez-

Pastrana, E., Vivo, A. \& Portela, A.

(2000). Influenza virus matrix protein is

the major driving force in virus budding.

Journal of Virology 74, 11538-11547.II

Latham, T. \& Galarza, J.M. (2001).

Formation of wild-type and chimeric

influenza virus-like particles following

simultaneous expression of only four

structural proteins. Journal of Virology

75, 6154-6165.II

Lohmeyer, J., Talens, L.T. \& Klenk, H.D.

(1979). Biosynthesis of the influenza

virus envelope in abortive infection.

Journal of General Virology 42, 73-88.II

Matassov, D., Cupo, A. \& Galarza, J.M.

(2007). A novel intranasal virus-like

particle (VLP) vaccine designed to

protect against the pandemic 1918

influenza A virus (H1N1). Viral

Immunology 20, 441-452.II

Middleton, D., Bingham, J., Selleck, P.,

Lowther, S., Gleeson, L., Lehrbacr ... [1] 
$421 \quad$ Figure legends

423 Figure 1. Triple recombinant baculovirus plasmid construct for expression of avian influenza H5N3 VLPs. Indicated are the polyhedrin promoter (PPH), p10 promoter (PP10), polyadenylation signal (SV40 pA and HSV tk pA), Tn7 regions (Tn7L and Tn7R), Ampicillin and Gentamicin gene resistance, influenza A/Duck/France/02166/2002 (H5N3) genes: HA (haemagglutinin) and NA (neuraminidase) proteins, and influenza A/Chicken/Italy/1067/99 (H7N1) gene: M matrix protein.

430 Figure 2. Immunofluorescence analyses of Sf9 cells infected with the triple recombinant baculovirus (bMH5N3-2). The HA, NA, and M1 expression was detected with anti-H5, anti-N3 and anti-M1 antibodies as described in materials and methods. Infected cells incubated with a SPF chicken serum (Cs SPF) were shown.

Figure 3. Purification of influenza proteins from Sf9 culture media.(A, B) Western blot analysis of fractions 6 to 12 from sucrose density gradient purification. Influenza virus A/Duck/France/02166/2002 (H5N3) was used as control (C+). The molecular weight of proteins was determined using Low Range Weight Marker (97.4 kD to $14 \mathrm{kD}$; Biorad). (A)

439 HA protein was detected using chicken specific serum H5N3/02166. (B) M1 protein was 440 detected using M1 specific murine monoclonal antibody (ATCC HB64). (C) Analysis of 441 fractions by neuraminidase inhibition assay. This test was carried out using chicken H2N3 antiserum for fractions and positive control, and chicken SPF antiserum for negative control. The homologous influenza virus A/Duck/Germany/1215/73 (H2N3) was used as control. 
446 Figure 4. Electron micrograph of negatively stained H5N3 avian influenza VLPs purified 447 through a 20-30-50\% sucrose density cushion from culture medium of Sf9 cells infected 448 with a triple recombinant baculovirus. Bar: $100 \mathrm{~nm}$.

449

450 Figure 5. SDS-PAGE of a sample of the 30-50\% sucrose interface purified VLPs stained 451 with Coomassie blue. Lane 1: $10 \mu$ l of purified VLPs. Lane 2: Low Range Weight Marker 452 (97.4 kD to $14 \mathrm{kD}$; Biorad).

454 Figure 6. Electron micrograph of immunogold-labelled H5N3 avian influenza VLPs purified through a 20-30-50\% sucrose density cushion from culture medium of Sf9 cells infected with a triple recombinant baculovirus. (A) VLPs were probed with anti-H5 antibody and counterstained with gold spheres coupled to anti-chicken IgG. (B) VLPs were probed with an anti-N3 antibody and counterstained as described above. Bar, 100nm. 
461 Table 1. Immunogenicity of VLPs for Muscovy ducks.

\begin{tabular}{lcc}
\hline & \multicolumn{2}{c}{ HI titre $\left(\log _{2}\right)^{\mathrm{a}}$} \\
\hline Group (5 ducks) & $\begin{array}{c}3 \text { weeks post-prime } \\
\text { (before boost) }\end{array}$ & 3 weeks post-boost \\
1 (unvaccinated) & $<2$ & $<2$ \\
$2(20 \mu \mathrm{g}$ VLPs) & $3.2(0.4)$ & $5.4(0.9)$ \\
$3(2 \mu \mathrm{g}$ VLPs) & $2.8(0.4)$ & $5.2(0.4)$ \\
\hline
\end{tabular}

$463{ }^{\text {a }}$ Geometric mean (standard deviation in parenthesis). The HI test was performed using 4

464 HA units of the inactivated A/duck/France/02166/2002(H5N3) AI strain. Positive results 465 are defined as $\geq 4 \log _{2}$. 
Page 18: [1] Deleted

a.prel

2/28/2008 3:03:00 PM

Brett, I.C. \& Johansson, B.E. (2005). Immunization against influenza A virus: comparison of conventional inactivated, live-attenuated and recombinant baculovirus produced purified hemagglutinin and neuraminidase vaccines in a murine model system. Virology 339, 273-280.

Bright, R.A., Carter, D.M., Crevar, C.J., Toapanta, F.R., Steckbeck, J.D., Cole, K.S., Kumar, N.M., Pushko, P., Smith, G., Tumpey, T.M. \& Ross, T.M. (2008). CrossClade Protective Immune Responses to Influenza Viruses with H5N1 HA and NA Elicited by an Influenza Virus-Like Particle. PLoS ONE 3, e1501.

Bright, R.A., Carter, D.M., Daniluk, S., Toapanta, F.R., Ahmad, A., Gavrilov, V., Massare, M., Pushko, P., Mytle, N., Rowe, T., Smith, G. \& Ross, T.M. (2007). Influenza virus-like particles elicit broader immune responses than whole virion inactivated influenza virus or recombinant hemagglutinin. Vaccine 25, 38713878.

Chen, B.J., Leser, G.P., Morita, E. \& Lamb R.A. (2007). Influenza virus hemagglutinin and neuraminidase, but not the matrix protein, are required for assembly and budding of plasmid-derived virus-like particles. Journal of Virology 81, 71117123.

Cherbonnel, M., Lamande, J., Allee, C., Schmitz, A., Ogor, K., Le Gall-Reculé, G., Le Bras, M.O., Guillemoto, C., Pierre, I., Picault, J.P. \& Jestin, V. (2007). Virologic findings in selected free-range mule duck farms at high risk for avian influenza infection. Avian Diseases 51, 408-413. 
Crawford, J., Wilkinson, B., Vosnesensky, A., Smith, G., Garcia, M., Stone, H. \& Perdue, M.L. (1999). Baculovirus-derived hemagglutinin vaccines protect against lethal influenza infections by avian H5 and H7 subtypes. Vaccine 17, 2265-2274.

Galarza, J.M., Latham, T. \& Cupo, A. (2005). Virus-like particle (VLP) vaccine conferred complete protection against a lethal influenza virus challenge. Viral Immunology 18, 244-251.

Gomez-Puertas, P., Albo, C., Perez-Pastrana, E., Vivo, A. \& Portela, A. (2000). Influenza virus matrix protein is the major driving force in virus budding. Journal of Virology 74, 11538-11547.

Latham, T. \& Galarza, J.M. (2001). Formation of wild-type and chimeric influenza virus-like particles following simultaneous expression of only four structural proteins. Journal of Virology 75, 6154-6165.

Lohmeyer, J., Talens, L.T. \& Klenk, H.D. (1979). Biosynthesis of the influenza virus envelope in abortive infection. Journal of General Virology 42, 73-88.

Matassov, D., Cupo, A. \& Galarza, J.M. (2007). A novel intranasal virus-like particle (VLP) vaccine designed to protect against the pandemic 1918 influenza A virus (H1N1). Viral Immunology 20, 441-452.

Middleton, D., Bingham, J., Selleck, P., Lowther, S., Gleeson, L., Lehrbach, P., Robinson, S., Rodenberg, J., Kumar, M. \& Andrew, M. (2007). Efficacy of inactivated vaccines against H5N1 avian influenza infection in ducks. Virology 359, 66-71.

Noad, R. \& Roy, P. (2003). Virus-like particles as immunogens. Trends in Microbiology 11, 438-444. 
OIE (2004). Highly pathogenic avian influenza. OIE manual of diagnostic tests and vaccines for terrestrial animals (mammals, birds, and bees). 5th ed. Paris, France.

Prel, A., Le Gall-Reculé, G., Cherbonnel, M., Grasland, B., Amelot, M. \& Jestin, V. (2007). Assessment of the protection afforded by triple baculovirus recombinant coexpressing H5, N3, M1 proteins against a homologous H5N3 low-pathogenicity avian influenza virus challenge in Muscovy ducks. Avian Diseases 51, 484-489.

Pushko, P., Tumpey, T.M., Bu, F., Knell, J., Robinson, R. \& Smith, G. (2005). Influenza virus-like particles comprised of the HA, NA, and M1 proteins of H9N2 influenza virus induce protective immune responses in BALB/c mice. Vaccine 23, 5751-5759.

Pushko, P., Tumpey, T.M., Van Hoeven, N., Belser, J.A., Robinson, R., Nathan, M., Smith, G., Wright, D.C. \& Bright, R.A. (2007). Evaluation of influenza virus-like particles and Novasome adjuvant as candidate vaccine for avian influenza. Vaccine 25, 4283-4290.

Quan, F.S., Huang, C., Compans, R.W. \& Kang, S.M. (2007). Virus-like particle vaccine induces protective immunity against homologous and heterologous strains of influenza virus. Journal of Virology 81, 3514-3524.

Roberts, P.C., Lamb, R.A. \& Compans, R.W. (1998). The M1 and M2 proteins of influenza A virus are important determinants in filamentous particle formation. Virology 240, 127-137.

Roy, P. (1996). Genetically engineered particulate virus-like structures and their use as vaccine delivery systems. Intervirology 39, 62-71. 
Steensels, M., Van Borm, S., Lambrecht, B., De Vriese, J., Le Gros, F.X., Bublot, M. \& van den Berg, T. (2007). Efficacy of an inactivated and a fowlpox-vectored vaccine in Muscovy ducks against an Asian H5N1 highly pathogenic avian influenza viral challenge. Avian Diseases 51, 325-331.

Sturm-Ramirez, K.M., Hulse-Post, D.J., Govorkova, E.A., Humberd, J., Seiler, P., Puthavathana, P., Buranathai, C., Nguyen, T.D., Chaisingh, A., Long, H.T., Naipospos, T.S., Chen, H., Ellis, T.M., Guan, Y., Peiris, J.S. \& Webster, R.G. (2005). Are ducks contributing to the endemicity of highly pathogenic H5N1 influenza virus in Asia? Journal of Virology 79, 11269-11279.

Swayne, D.E., Beck, J.R. \& Kinney, N. (2000). Failure of a recombinant fowl poxvirus vaccine containing an avian influenza hemagglutinin gene to provide consistent protection against influenza in chickens preimmunized with a fowl pox vaccine. Avian Diseases 44, 132-137.

van den Berg, T., Lambrecht, B., Marche, S., Steensels, M., Van Borm, S. \& Bublot, M. (2008). Influenza vaccines and vaccination strategies in birds. Comparative Immunology Microbiology and Infectious Disease 31, 121-165.

van Deusen, R.A., Hinshaw, V.S., Senne, D.A. \& Pellacani, D. (1983). Micro neuraminidase-inhibition assay for classification of influenza A virus neuraminidases. Avian Diseases 27, 745-750.

van Oers, M.M. (2006). Vaccines for viral and parasitic diseases produced with baculovirus vectors. Advance in Virus Research 68, 193-253. 


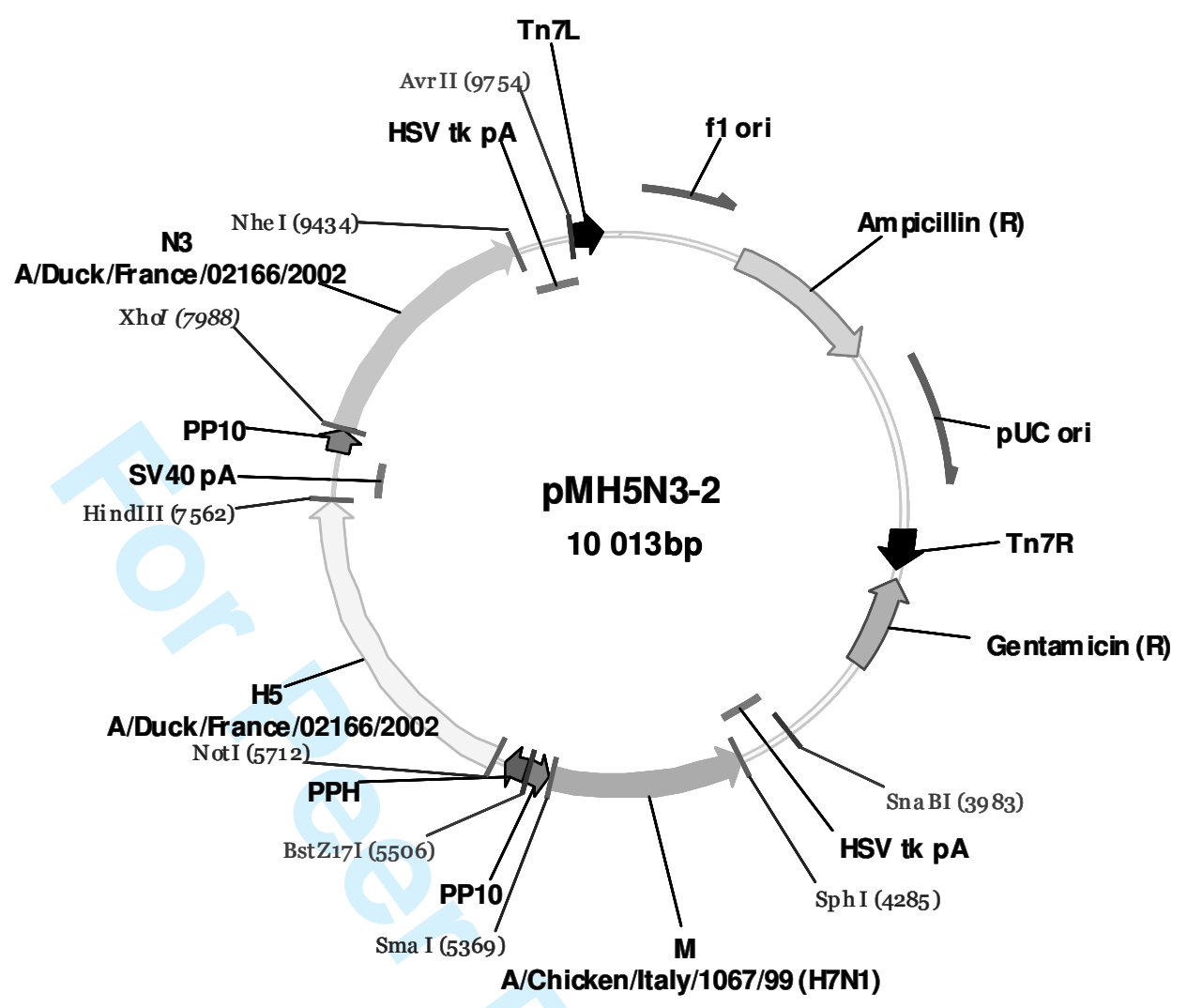

Figure 1. Triple recombinant baculovirus plasmid construct for expression of avian influenza H5N3 VLPs. Indicated are the polyhedrin promoter (PPH), p10 promoter (PP10), polyadenylation signal (SV40 pA and HSV tk pA), Tn7 regions (Tn7L and Tn7R), Ampicillin and Gentamicin gene resistance, Influenza A/Duck/France/02166/2002 (H5N3) genes: HA (Hemagglutinin) and NA (Neuraminidase) proteins, and Influenza A/Chicken/Italy/1067/99 (H7N1) gene: M matrix protein. 


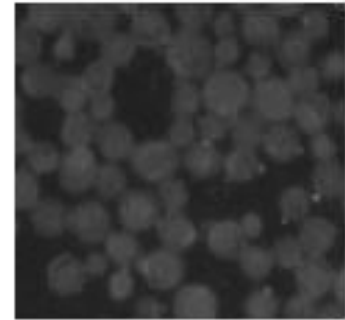

cS SPF

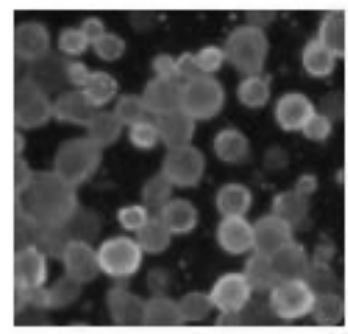

N3 - specific antibody

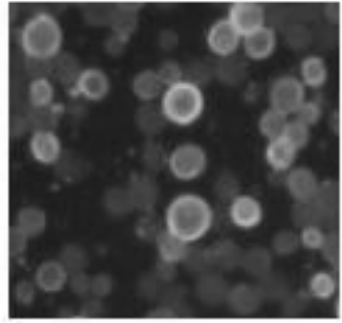

H5 - specific antibody

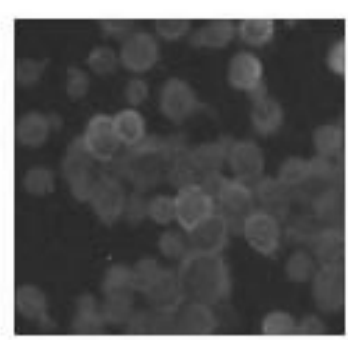

Ml- specific antibody

Figure 2. Immunofluorescence analyses of Sf9 cells infected with the triple recombinant baculovirus (bMH5N3-2). The HA, NA, and M1 expression was detected with anti-H5, anti$\mathrm{N} 3$ and anti-M1 antibodies as described in materials and methods. Infected cells incubated with a SPF chicken serum (Cs SPF) were shown. 
(A)
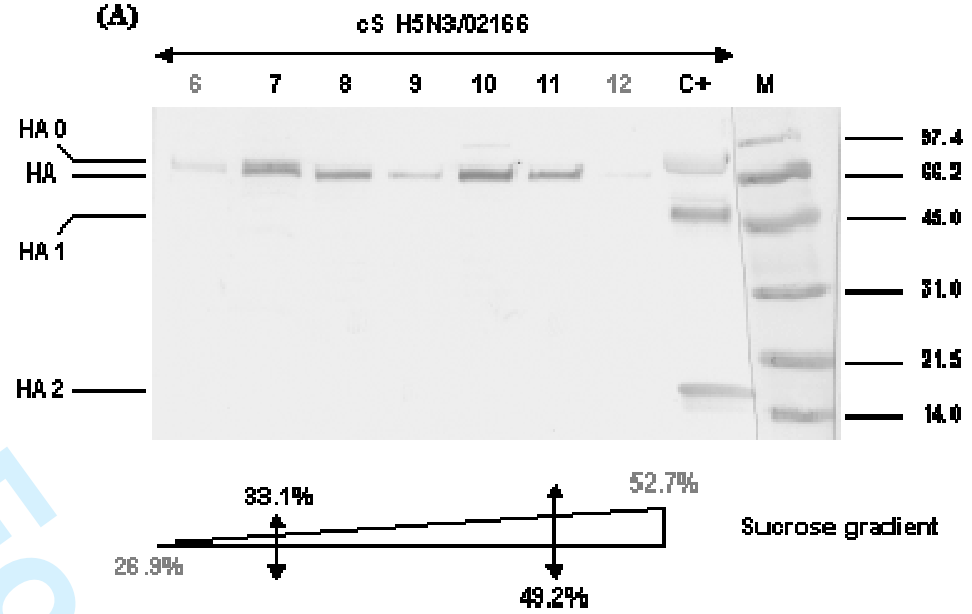

(B) W1 speoifio murine monoclonal antibody
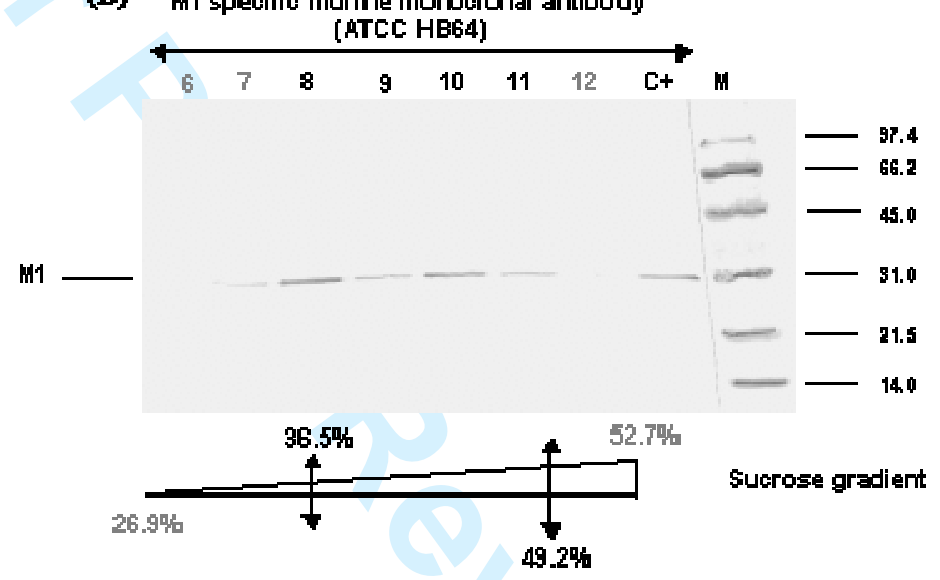

(C)

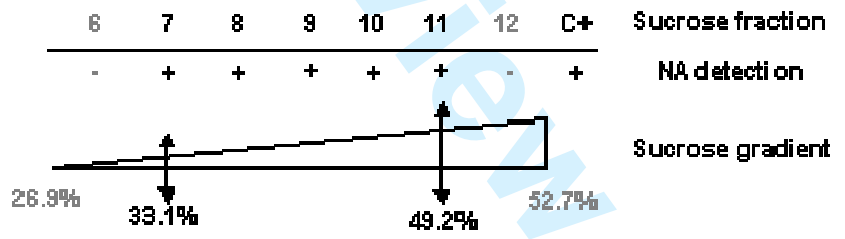

Figure 3. Purification of influenza proteins from Sf9 culture media.(A, B) Western blot analysis of fractions 6 to 12 from sucrose density gradient purification. Influenza virus A/Duck/France/02166/2002 (H5N3) was used as control (C+). The molecular weight of proteins was determined using Low Range Weight Marker (97.4 kD to $14 \mathrm{kD}$; Biorad). (A) HA protein was detected using chicken specific serum H5N3/02166. (B) M1 protein was 
detected using M1 specific murine monoclonal antibody (ATCC HB64). (C) Analysis of fractions by neuraminidase inhibition assay. This test was carried out using chicken H2N3 antiserum for fractions and positive control, and chicken SPF antiserum for negative control. The homologous influenza virus A/Duck/Germany/1215/73 (H2N3) was used as control. 


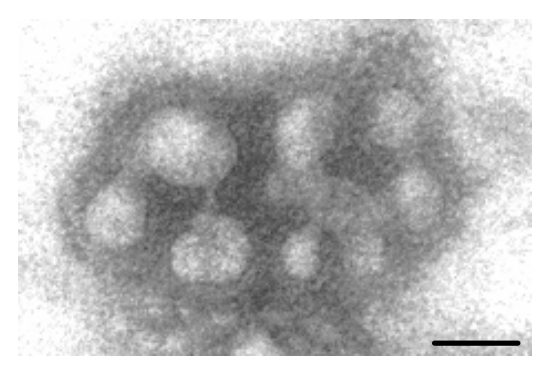

Figure 4. Electron micrograph of negatively stained H5N3 avian influenza VLPs purified through a 20-30-50\% sucrose density cushion from culture medium of Sf9 cells infected with the triple recombinant baculoviruses. Bar: $100 \mathrm{~nm}$. 


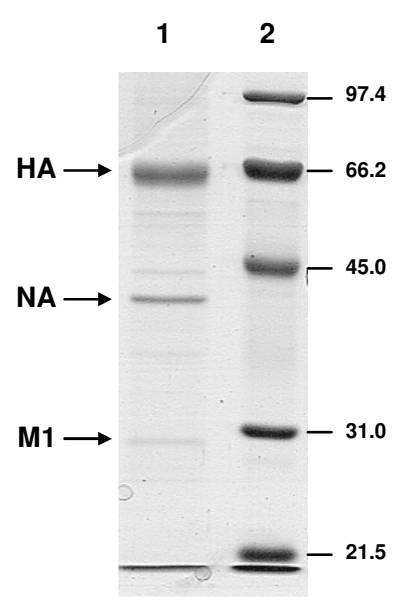

Figure 5. SDS-PAGE of a sample of the 30-50\% sucrose interface purified VLPs stained with Coomassie blue. Lane 1: $10 \mu 1$ of purified VLPs. Lane 2: Low Range Weight Marker (97.4 $\mathrm{kD}$ to $14 \mathrm{kD}$; Biorad). 

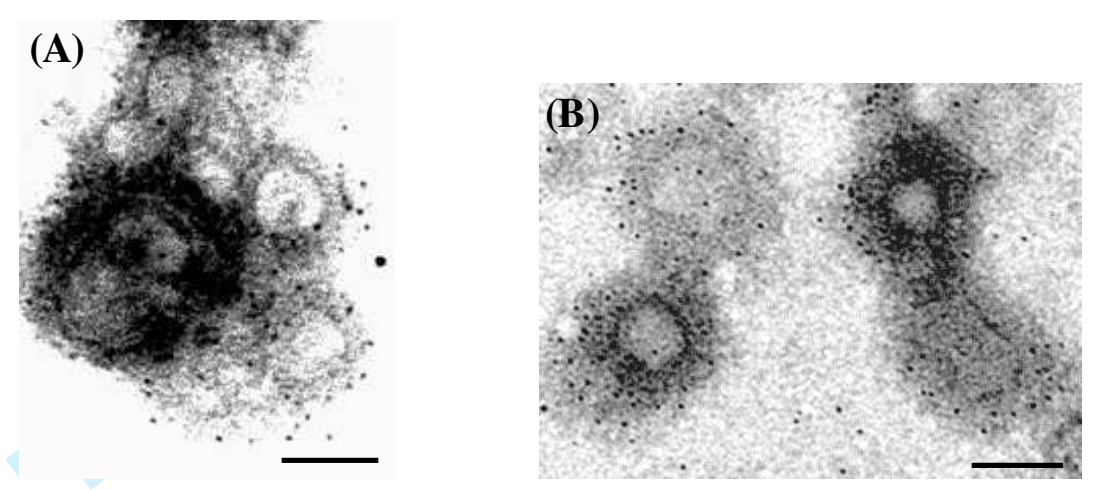

Figure 6. Electron micrograph of immunogold-labeled H5N3 avian influenza VLPs purified through a 20-30-50\% sucrose density cushion from culture medium of Sf9 cells infected with the triple recombinant baculoviruses. (A) VLPs were probed with anti-H5 antibody and counterstained with gold spheres coupled to anti-chicken IgG. (B) VLPs were probed with an anti-N3 antibody and counterstained as described above. Bar, 100nm. 